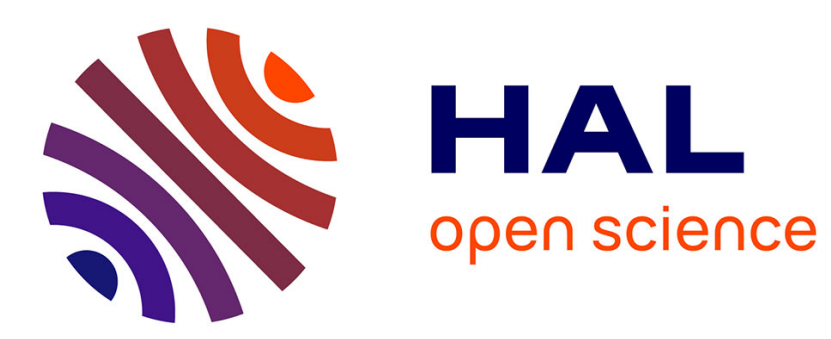

\title{
Effect of frictional anisotropy on the quasistatic motion of a deformable solid sliding on a planar surface
} Zhi-Qiang Feng, Mohammed Hjiaj, Géry de Saxcé, Zenon Mróz

\section{To cite this version:}

Zhi-Qiang Feng, Mohammed Hjiaj, Géry de Saxcé, Zenon Mróz. Effect of frictional anisotropy on the quasistatic motion of a deformable solid sliding on a planar surface. Computational Mechanics, 2006, 37 (4), pp.349-361. 10.1007/s00466-005-0674-5 . hal-01179152

\section{HAL Id: hal-01179152 \\ https://hal.science/hal-01179152}

Submitted on 30 Jun 2018

HAL is a multi-disciplinary open access archive for the deposit and dissemination of scientific research documents, whether they are published or not. The documents may come from teaching and research institutions in France or abroad, or from public or private research centers.
L'archive ouverte pluridisciplinaire HAL, est destinée au dépôt et à la diffusion de documents scientifiques de niveau recherche, publiés ou non, émanant des établissements d'enseignement et de recherche français ou étrangers, des laboratoires publics ou privés. 


\section{Effect of frictional anisotropy on the quasistatic motion of a deformable solid sliding on a planar surface}

\begin{abstract}
In this paper, the motion of a deformable body sliding on a half-plane is considered. The solid undergoes large displacements but small strains. An orthotropic friction model described by an elliptic cone is considered. This model allows to describe the slidingdirection dependence of the frictional behavior observed in experience. The algorithm used to solve the problem is based on a weak variational statement of the frictional contact law. The Uzawa algorithm is used to solve the discrete problem. The corresponding algorithm is robust and can deal with large sliding increments. The study shows that frictional properties can influence significantly the trajectory of a deformable body sliding on a frictional surface.
\end{abstract}

Keywords Elliptic friction criterion · Large sliding $\cdot$ Variational formulation $\cdot$ Uzawa algorithm

\section{Z. -Q. Feng}

Laboratoire de Mécanique d'Evry,

Université d'Evry-Val d'Essonne, 40 rue du Pelvoux, 91020 Evry Cedex, France

M. Hjiaj ( $\varangle)$

Department of Civil Engineering, The University of Newcastle, Callaghan, NSW, 2308, Australia

E-mail: mohammed.hjiaj@insa-rennes.fr

Tel.: + 33-2-23238711

Fax: + 33-2-23238448

Present address: M. Hjiaj

INSA de Rennes, 20 avenue des Buttes de Coësmes,

35043 Rennes cedex, France

G. de. Saxcé

Laboratoire de Mécanique de Lille CNRS/UMR 8107, Boulevard Paul Langevin, F-59655 Villeneuve d'Ascq Cedex, France

Z. Mróz

Polish Academy of Sciences,

Institute of Fundamental Technological Research,

Świętokrzyska 21, 00-049 Warsaw, Poland

\section{Introduction}

Planning the motion of a body in contact with a planar surface is an usual task for a robotic system. The goal of planning is to determine force commands which, if executed, would achieve a pre-specified motion of the body. If the task is to be done as quickly as possible, dynamic effects must be included. Otherwise, a quasistatic model, one which ignores inertial forces, may prove a good alternative. Models used in robotics assume that the pushed body is rigid so the motion cannot always be uniquely predicted. However, if the body is assumed deformable, the static indeterminacy is removed which makes the solution of the problem easy. An accurate description of the body motion cannot be achieved unless an adequate friction model is used. Though, usually assumed to be constant, the friction coefficient often varies with the sliding direction. So the usual isotropic Coulomb's model is a gross approximation and more complex models have to be introduced. In what follows, the effect of the friction model on the motion of an object being pushed over a floor is investigated.

A friction model is defined by the friction condition which specifies a convex set of admissible contact forces and the sliding rule which stipulates what directions of sliding are allowed. The shape of the friction surface and the nature of slip rule have a fairly strong influence on the motion of sliding objects. In most studies, the limit surface is used under its isotropic form. As a result, the frictional behavior is independent of the sliding direction. However this assumption seems to be unrealistic. Indeed many experimental studies show that the frictional behavior can change drastically with the sliding direction, requiring an anisotropic model. This anisotropy can be attributed to two main sources. The first one is the anisotropy of the material constituting the solid and the planar surface that manifest themselves on the contact surface as well. The second one is technological. The industrial process used to fabricate the bodies can 
create striations along preferential directions. In fact, most machining, finishing and superfinishing operations are directional, and machined surfaces have particular striation patterns unique to type of machining. Also specific techniques of manufacture produce a surface with anisotropic frictional properties. For a large number of machining processes, the striation directions are mutually orthogonal. For such surfaces, an orthotropic frictional model will provide a better description of the frictional behavior.

A common way to model frictional anisotropy is to adopt an elliptic friction condition in the plane $r_{n}=$ const, where $r_{n}$ is the contact pressure. In the sequel, the term "contact pressure" means the normal component of the contact stress vector which has a tangential component when friction exists on the contact surface. Two friction coefficients, one in the $x$-direction and another in the $y$-direction (orthogonal to the $x$-direction), have to be determined. These coefficients correspond to the ellipse semi-axes for $r_{n}=1$. It will be shown that frictional anisotropy has a significant impact for problems such as motion of sliding objects. For a specified pushing force the trajectory of an object depends on the frictional properties of the planar surface.

In this paper, the motion of a pushed deformable block sliding on a frictional planar surface is studied. The block is assumed to be elastic. Large displacements are allowed but the block is assumed to undergo small strain. The supporting surface has anisotropic frictional properties. The problem is solved in a quasi-static fashion using the finite element method and the total lagrangian technique. The algorithm used to solve the problem is based on a variational formulation of the frictional contact law. In the next section, contact variables are defined and the unilateral contact law is presented. For bodies in contact, this law can be written in a rate form where the kinematical variable is the velocity. Section 3 is concerned with anisotropic friction models with convex limit friction surfaces of elliptic shape. In Sect. 4, the rate form of the Signorini conditions are coupled with the sliding rule to give the complete frictional contact law for bodies in contact. A formulation based on a variational inequality is presented at the end of the section. Section 5 deals with the governing discrete relations of the considered frictional contact model. In
Sect. 6, the discretized frictional contact problem is outlined and the F.E. algorithm is presented in Sect. 8. The example shown in Sect. 9 highlights the influence of the frictional model on the motion of sliding objects.

\section{Deformable block sliding on a planar surface - Governing equations}

The pushed block undergoes large displacements which require an appropriate setting of the problem. The basics of continuum contact mechanics in the large displacement setting are briefly addressed in this section. The details are omitted as they are not necessary for the current study (see, [4]).

\subsection{Kinematics and statics}

Consider a deformable block $\mathscr{B}$ undergoing large displacements and small strains (Fig. 1). The body $\mathscr{B}$ occupies in a referential configuration the open, simply connected, bounded domain $\Omega \subset \mathbb{R}^{3}$. The closure $\bar{\Omega}$ of $\Omega$ represents the reference configuration of $\mathscr{B}$ which corresponds to the initial one in the total Lagrangian formulation, adopted here. A material particle $\mathscr{M}$ in $\Omega$ is identified by its reference position vector $\mathbf{X}$. The successive deformed configurations $\mathscr{B}^{t}, t \in[0, T]$, are defined by a differentiable and invertible mapping $\varphi$ :

$\varphi(\mathbf{X}, t): \bar{\Omega} \times[0, T] \rightarrow \mathbb{R}^{3}$,

where $\mathbf{I}=:[0, T]$ is the time domain of interest, corresponding to the loading process. The time $t \in \mathbf{I}$ characterizing the reference configuration is $t_{0}=0$. The position of a particle of the body in the current configuration $\mathscr{B}^{t}$ is defined using the current coordinate vector $\mathbf{x}=\varphi(\mathbf{X}, t)$. The displacement of a material point from the initial configuration is the difference between its current and its initial position

$\mathbf{u}=\varphi(\mathbf{X}, t)-\mathbf{X}$

The velocity of a material point in the reference configuration is given by

$\dot{\mathbf{u}}(\mathbf{X}, t)=\frac{\partial \varphi}{\partial t}(\mathbf{X}, t)$
Fig. 1 Block sliding on a frictional surface

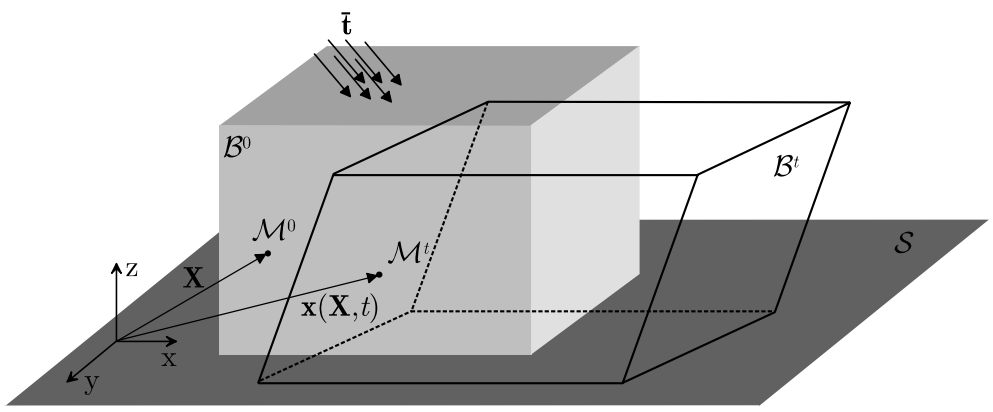


The boundary $\Gamma(t)$ of $\mathscr{B}^{t}$ is assumed to be sufficiently smooth everywhere such that an outward unit normal vector, denoted by $\mathbf{n}$, can be defined everywhere on $\Gamma(t)$. To fix the ideas, the body under consideration is a cube in frictional contact with a planar surface, denoted $\mathscr{S}$. We assume that the boundary $\Gamma(t)$ of $\mathscr{B}^{t}$ can be divided into three disjoint parts: $\Gamma_{u}(t)$ with prescribed displacements, $\Gamma_{t}(t)$ with prescribed boundary loads, and the potential contact surface $\Gamma_{c}(t)$ where $\mathscr{B}_{t}$ may possibly come into contact with the half-plane at some time $t$ :

$\Gamma(t)=\Gamma_{u}(t) \cup \Gamma_{t}(t) \cup \Gamma_{c}(t)$

Displacements are imposed on the upper face of the cube and the lower face corresponds to the contact surface $\Gamma_{c}$. The remaining faces are stress free. The transformation gradient $\mathbf{F}$ relates the current configuration to the reference configuration by

$\mathbf{F}(\mathbf{X}, t)=\frac{\partial \mathbf{x}(\mathbf{X}, t)}{\partial \mathbf{X}}=\mathbf{I}+\frac{\partial \mathbf{u}(\mathbf{X}, t)}{\partial \mathbf{X}}, \quad \operatorname{det} \mathbf{F}>0$

The space $\mathscr{U}_{a d}$ defined by

$\mathscr{U}_{a d}=\left\{\mathbf{u} \in \mathscr{U} \mid \operatorname{det}(\mathbf{I}+\nabla \mathbf{u})>0 \forall \mathbf{X} \in \Omega\right.$ and $\left.\mathbf{u}=\overline{\mathbf{u}} \forall \mathbf{X} \in \Gamma_{u}\right\}$

contains all admissible displacement fields that satisfy kinematic relations. The strain measure adopted here is the Green-Lagrange strain tensor $\mathbf{E}$ which refers to the initial configuration $\mathscr{B}_{0}$

$\mathbf{E}=\frac{1}{2}(\mathbf{C}-\mathbf{I})=\frac{1}{2}\left(\nabla \mathbf{u}+(\nabla \mathbf{u})^{\mathrm{T}}+(\nabla \mathbf{u})^{\mathrm{T}} \nabla \mathbf{u}\right)$

where $\mathbf{C}=\mathbf{F}^{\mathrm{T}} \mathbf{F}$ is the right Cauchy-Green tensor.

The strong form of the equilibrium equations in the reference configuration is given by

$\operatorname{Div} \mathbf{P}+\overline{\mathbf{f}}_{0}=0 \quad$ in $\Omega(0)$

$\mathbf{P n}_{0}=\mathbf{t}_{0} \quad$ on $\Gamma_{t}(0)$

where $\mathbf{P}$ denotes the first Piola-Kirchhoff stress tensor, $\overline{\mathbf{f}}_{0}=J \overline{\mathbf{f}}$ the body forces per unit undeformed volume, $\mathbf{n}_{0}$ the normal to $\Gamma_{t}(0)$ and $\overline{\mathbf{t}}$ the imposed surface tractions. The body $\mathscr{B}$ is modelled using the linear St Venant constitutive relation where the Green-Lagrange strains are related to the second Piola-Kirchhoff stresses by

$\mathbf{S}=\mathbf{F}^{-1} \mathbf{P}=\frac{\partial W(\mathbf{E})}{\partial \mathbf{E}}$

with $W(\mathbf{E})$ being the energy density function (quadratic).

\subsection{Contact mechanics}

In the initial configuration $\mathscr{B}_{0}$, the lower face of the block is in full contact with the half-plane. The external loading starts with with a uniform vertical motion of the upper face which compress the block so it remains in contact with the planar surface during sliding. The sliding motion is obtained by imposing a uniform horizontal displacement to the upper surface. During the motion of the block, contact conditions must be fulfilled at each time-instant and material points in contact with $\mathscr{S}$ in the initial configuration $\mathscr{B}_{0}$ must not cross the halfplane. Accordingly, contact conditions must be formulated in the current configuration $\mathscr{B}_{t}$. On the surface $\mathscr{S}$, an orthogonal vector $\mathbf{n}$ directed towards $\mathscr{B}$ is defined (Fig. 2). The unit normal $\mathbf{n}$ coincide here with the unit vector associated with the $z$-axis of the coordinate system. Within the half-plane $\mathscr{S}$ two unit vectors $\mathbf{t}_{x}$ and $\mathbf{t}_{y}$ are defined such that with $\mathbf{n}$, they form an orthogonal local basis. Several options are possible for setting up the base vectors, depending on a particular choice of the unit tangent vectors. A natural choice for the unit tangent vectors is the principal orthotropy directions. The relative slip velocity corresponds to the block velocity $\dot{\mathbf{u}}$ since the planar surface is motionless. As a result of the external loading a contact force distribution equilibrating the loads is developed. The surface traction vector $\mathbf{r}^{*}$ at a material point $\mathscr{M} \in \Gamma_{c}$ is given by

$\mathbf{r}^{*}=\mathbf{P} \mathbf{n}_{0}$

The contact force distribution $\mathbf{r}^{*}$ acts on the half-plane $\mathscr{S}$. According to the principle of action and reaction, the block $\mathscr{S}$ is subjected to

$\mathbf{r}=-\mathbf{P} \mathbf{n}_{0}$

In the local coordinate system defined by the plane $\mathscr{S}$ and the normal $\mathbf{n}$, any variable $\dot{\mathbf{u}}$ or $\mathbf{r}$ may be uniquely decomposed into normal and tangential components according to

$\dot{\mathbf{u}}=\dot{\mathbf{u}}_{t}+\dot{u}_{n} \mathbf{n} \quad \dot{\mathbf{u}}_{t} \in \mathscr{S}, \dot{u}_{n} \in \mathbb{R}$

$\mathbf{r}=\mathbf{r}_{t}+r_{n} \mathbf{n} \quad \mathbf{r}_{t} \in \mathscr{S}, r_{n} \in \mathbb{R}$

The frictional force $\mathbf{r}_{t}$ are dissipative and always opposes sliding (see Fig. 2). To ensure that the dissipation is positive the velocity $\dot{\mathbf{u}}$ will be preceded by a sign "minus" $(-\dot{\mathbf{u}})$. The unilateral contact condition requires that material points $\mathbf{X} \in \Gamma_{c}$ are either in contact or not in contact with the plane $\mathscr{S}$. Therefore, the body $\mathscr{B}$ is allowed to separate but not to cross the plane $\mathscr{S}$. This condition constraints the placement of the body $\mathscr{B}$. In the problem under consideration, the lower face of the cube remain in contact with the planar surface during the whole loading process. Using the above decomposition and taken into account that the initial gap (distance between the block and the planar surface projected

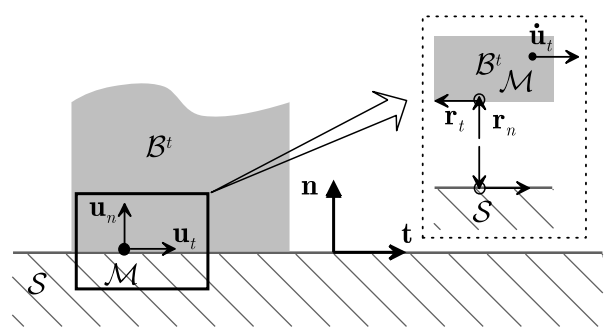

Fig. 2 Kinematics of contact 
on the normal) is null, the non-penetration condition can be expressed by

$-u_{n} \leq 0$

A dual relation involves the contact pressure $r_{n}$ acting on the block which must be positive $\left(r_{n} \geq 0\right)$ where there is contact and zero where there is no contact. This condition is often referred to the non-adhesion condition. This set of relations may be summarized by the so-called Signorini conditions:

$-u_{n} \leq 0 \quad r_{n} \geq 0 \quad u_{n} r_{n}=0$

which has to be satisfied at each time-instant $t \in \mathbf{I}$. In the case of persistent contact $\left(u_{n}=0\right)$, the unilateral contact law can be formulated in a rate form:

$-\dot{u}_{n} \leq 0 \quad r_{n} \geq 0 \quad \dot{u}_{n} r_{n}=0 \quad$ on $\Gamma_{c}$

The relation (12.a) imposes that contacting bodies must either remain in contact $\left(\dot{u}_{n}=0\right)$ or must separate $\left(\dot{u}_{n}>0\right)$ (only a loss of contact is allowed). The rate formulation of the Signorini conditions (12) can be combined with the sliding rule to derive the full frictional contact law applicable to material points of $\Gamma_{c}$ already in contact. This complete law specifies allowable velocities of these points such that impenetrability, non-adhesion and the sliding rule are satisfied. In a more general situation, a positive gap may appear $\left(u_{n}>0\right)$. In that case, the normal relative velocity is arbitrary $\left(\dot{u}_{n} \in \mathbb{R}\right)$ and the normal reaction force is equal to zero $\left(r_{n}=0\right)$. The equations developed above need to be completed by a set of relations specifying the friction model and the slip rule.

\section{Orthotropic friction model}

A rate-independent friction model is considered where a linear dependence of the limit tangential force on the normal force holds. A theoretical investigation on friction surfaces and sliding rules has been carried out by Michałowski and Mróz [2]. Their study, based on a model of rigid anisotropic asperities, shows that cross sections of the friction cone could be slightly non-convex. However they can be approximated accurately by ellipses. A family of anisotropic friction models, described by a friction condition of elliptic shape (in the plane $r_{n}=$ const) is considered. The principal axes of the ellipse coincide with the orthotropy axes $x$ and $y$.

\subsection{Friction criterion}

The asperity model used by Michałowski and Mróz ([2]) to study anisotropic frictional contact phenomenon generates limit friction curves that can be accurately approximated by ellipses. The generic form of such convex friction criterion is given by

$f\left(r_{t_{x}}, r_{t_{y}}, r_{n}\right)=\left\|\mathbf{r}_{t}\right\|_{\mu}-r_{n}=0$

where $\|\bullet\|_{\mu}$ denotes the elliptic norm

$\left\|\mathbf{r}_{t}\right\|_{\mu}=\sqrt{\left(\frac{r_{t_{x}}}{\mu_{x}}\right)^{2}+\left(\frac{r_{t_{y}}}{\mu_{y}}\right)^{2}}$

The coefficients $\mu_{x}$ and $\mu_{y}$ are the principal friction coefficients. The curve (13) intersects the $x$-axis at $\mu_{x} r_{n}$ and $-\mu_{x} r_{n}$; it intersects the $y$-axis at $\mu_{y} r_{n}$ and $-\mu_{y} r_{n}$ (Fig. $3)$. Introducing the friction coefficients matrix $\mathbb{M}$, defined by

$\mathbb{M}=\left[\begin{array}{cc}\mu_{x} & 0 \\ 0 & \mu_{y}\end{array}\right]$,

the elliptic norm (14) used in the friction criterion definition (13) can be replaced by the usual Euclidean norm $\|\bullet\|$ :

$\left\|\mathbf{r}_{t}\right\|_{\mu}=\left\|\mathbb{M}^{-1} \mathbf{r}_{t}\right\|$

The classical isotropic Coulomb's friction criterion is recovered by setting

$\mu_{x}=\mu_{y}=\mu$

so $\mathbb{M}$ is the unit matrix. The set $K_{\mu}$ of allowable contact forces $\mathbf{r}$, defined by
Fig. 3 Elliptic friction condition

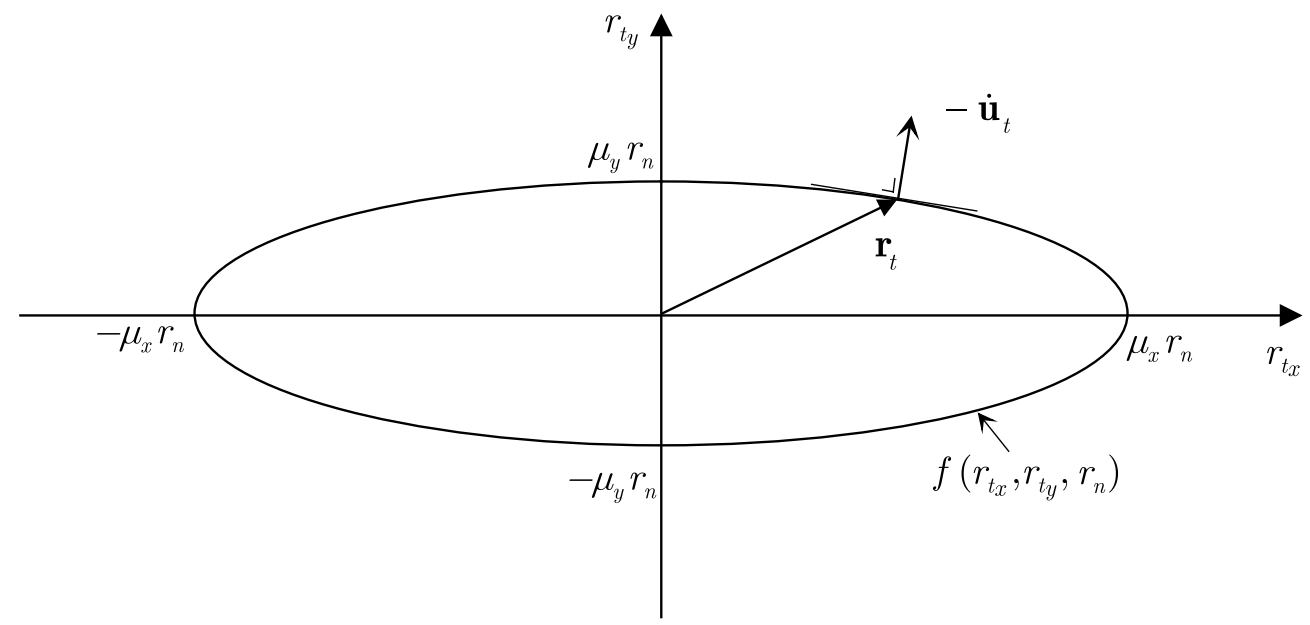


$K_{\mu}=\left\{\mathbf{r} \in \mathbb{R}^{3} \mid\left\|\mathbf{r}_{t}\right\|_{\mu}-r_{n} \leq 0\right\}$,

is convex. Its boundary and interior are denoted "bd $K_{\mu}$ " and "int $K_{\mu}$ ", respectively. Now it is appropriate to introduce the cone $K_{\mu}^{*}$ dual (or polar) to $K_{\mu}$. This set will be used later in the paper. By definition the polar cone $K_{\mu}^{*}$ is the set comprising all vectors $\mathbf{v} \in \mathbb{R}^{3}$ satisfying the following inequality

$\mathbf{v} \in \mathbb{R}^{3}, \quad \mathbf{r} \cdot \mathbf{v} \leq 0, \quad \forall \mathbf{r} \in K_{\mu}$

where the dot "." stands for the usual scalar product. The scalar product in (16) is developed in the following manner

$$
\begin{aligned}
\mathbf{r} \cdot \mathbf{v} & =r_{t_{x}} v_{t_{x}}+r_{t_{y}} v_{t_{y}}+r_{n} v_{n} \\
& \leq\left\|\mathbf{r}_{t}\right\|_{\mu}\left\|\mathbf{v}_{t}\right\|_{\mu}^{*}+r_{n} v_{n} \\
& \leq r_{n}\left(\left\|\mathbf{v}_{t}\right\|_{\mu}^{*}+v_{n}\right)
\end{aligned}
$$

where the Cauchy-Schwartz inequality has been used to obtain the first inequality and the friction criterion (15) has been used to obtain the second one. The normal reaction $r_{n}$ being positive, the inequality (19) is satisfied if

$\left\|\mathbf{v}_{t}\right\|_{\mu}^{*}+v_{n} \leq 0$

where the norm $\|\bullet\|_{\mu}^{*}$, dual of $\|\bullet\|_{\mu}$, is given by

$\left\|\mathbf{v}_{t}\right\|_{\mu}^{*}=\sqrt{\left(\mu_{x} v_{t_{x}}\right)^{2}+\left(\mu_{y} v_{t_{y}}\right)^{2}}=\left\|\mathbb{M} \mathbf{v}_{t}\right\|$

All vectors $\mathbf{v}$ satisfying (20) belongs to $K_{\mu}^{*}$ :

$K_{\mu}^{*}=\left\{\mathbf{v} \in \mathbb{R}^{3} \mid\left\|\mathbf{v}_{t}\right\|_{\mu}^{*}+v_{n} \leq 0\right\}$

\subsection{Slip rule}

An associated sliding rule (in the plane $r_{n}=$ cte) is adopted where the direction of sliding (up to the sign ) is given by the gradient to the friction cone and its magnitude by the multiplier $\dot{\lambda}$ :

$-\dot{u}_{n}=0$

$-\dot{u}_{t_{x}}=\dot{\lambda} \frac{\partial f}{\partial r_{t_{x}}}=\frac{\dot{\lambda}}{\mu_{x}^{2}} \frac{r_{t_{x}}}{\left\|\mathbf{r}_{t_{1}}\right\|_{\mu}}$

$-\dot{u}_{t_{y}}=\dot{\lambda} \frac{\partial f}{\partial r_{t_{y}}}=\frac{\dot{\lambda}}{\mu_{y}^{2}} \frac{r_{t_{y}}}{\left\|\mathbf{r}_{t}\right\|_{\mu}}$

The multiplier $\dot{\lambda}$ is required to satisfy the complementarity relations

$f\left(\mathbf{r}_{t}, r_{n}\right) \leq 0, \quad \dot{\lambda} \geq 0, \quad \dot{\lambda} f\left(\mathbf{r}_{t}, r_{n}\right)=0$

The components of the tangential reaction can be eliminated from the slip rule (24-25) so the expression of $\dot{\lambda}$ as function of $-\dot{\mathbf{u}}_{t}$ is obtained

$\dot{\lambda}=\left\|-\dot{\mathbf{u}}_{t}\right\|_{\mu}^{*}$

The inverse of the relationships (23-25) is

$$
\begin{aligned}
& r_{n}>0 \\
& r_{t_{x}}=r_{n} \frac{\mu_{x}^{2}\left(-\dot{u}_{t_{x}}\right)}{\left\|-\dot{\mathbf{u}}_{t}\right\|_{\mu}^{*}} \\
& r_{t_{y}}=r_{n} \frac{\mu_{y}^{2}\left(-\dot{u}_{t_{y}}\right)}{\left\|-\dot{\mathbf{u}}_{t}\right\|_{\mu}^{*}}
\end{aligned}
$$

\section{The frictional contact law}

The frictional contact law aims to describe contact interactions. We consider now the previous friction law embedding the impenetrability condition for completeness. The sliding rule is combined with the rate form of the unilateral contact conditions to obtain the frictional contact law. The multivalued nature of this strongly nonlinear law makes problems involving frictional contact among the most difficult ones in solid mechanics.

\subsection{Analytical formulation}

The complete form of the frictional contact law deals with the three possible physical situations, which are separation, contact with sticking, and contact with sliding. Dissipation occurs only for the last case. This law is applicable only to material points in contact. Two overlapped "if ... then ... else" statements can be used to write it analytically (Box 1):

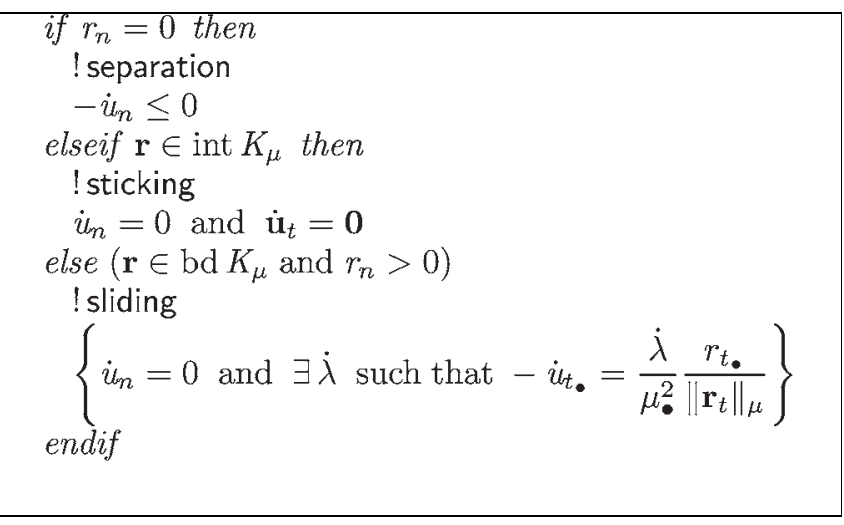

In the first and the second part of the statement, the multivalued character of the frictional contact constitutive model is revealed. If $r_{n}$ is null then the velocity $\dot{\mathbf{u}}$ is arbitrary but its normal component $\dot{u}_{n}$ should be positive. In others words, an infinite number of velocity vectors $\dot{\mathbf{u}} \in \mathbb{R}^{3}$ can be related to one element of $\mathbf{r} \in \mathbb{R}^{3}$. On the other hand, if $-\dot{\mathbf{u}}$ is null then the reaction $\mathbf{r}$ should be in $K_{\mu}$ but its direction or magnitude are not specified. They are arbitrary. Again, one element of $\mathbb{R}^{3}$ 
$(-\dot{\mathbf{u}}=\mathbf{0})$ can be related to an infinite number of $\mathbf{r} \in \mathbb{R}^{3}$. The inverse constitutive frictional contact law, i.e. the relationship $\mathbf{r}(-\dot{\mathbf{u}})$ can be written as (Box 2):

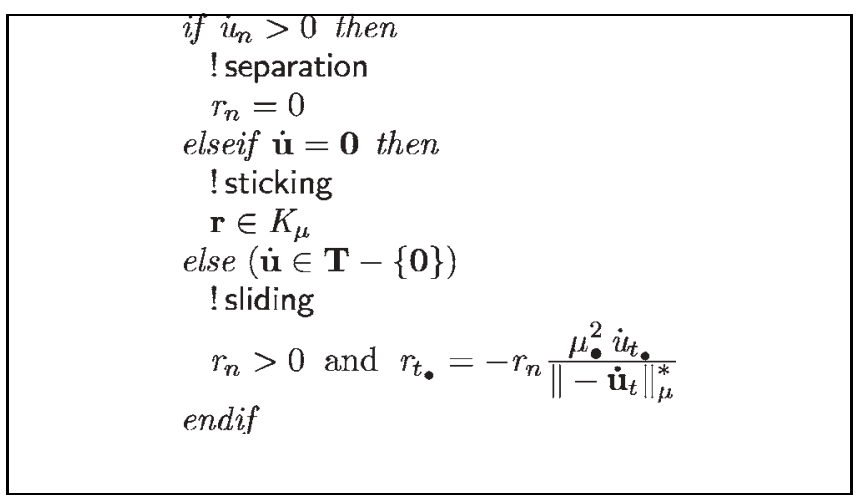

Similar considerations regarding the multi-valued character of the relatioship can be drawn for the inverse law.

\subsection{Variational inequality-based formulation}

The previous forms of the frictional contact law, given in Box 1 and Box 2, are well suited for classical numerical implementations where the contact problem is dissociated from the friction problem. However on the contact surface, contact cannot be separated from friction. Therefore a coupled algorithm that solved both problems in one step could improve the computational performances and robustness. With this aim in mind, the frictional contact law need to be reformulated. The main idea behind this reformulation is to provide a natural basis for the application of the Uzawa algorithm.

The slip rule, as written in relations (23-25), exhibits a structure similar to the non-associated flow rule in plasticity. Indeed, during sliding, contact is maintained. Therefore the normal velocity is equal to zero $\left(\dot{u}_{n}=0\right)$ and not related to the normal component of the reaction $r_{n}$ through normality. In fact, if we regard the contact force $\mathbf{r}$ and the velocity $-\dot{\mathbf{u}}$ as conjugate quantities of each other, the normality will not occur since it would require that the velocity would have a normal separating component.

To avoid complex notations due to elliptic norms, the variational-inequality based formulation is derived for the isotropic model $\mu_{x}=\mu_{y}$. The sliding rule is given by

$$
-\dot{u}_{n}=0, \quad-\dot{\mathbf{u}}_{t}=\dot{\lambda} \frac{\partial f(\mathbf{r})}{\partial \mathbf{r}_{t}}
$$

but can be rewritten in the following obvious way:

$-\left(\dot{u}_{n}+\mu \dot{\lambda}\right)=\dot{\lambda} \frac{\partial f}{\partial r_{n}}, \quad-\dot{\mathbf{u}}_{t}=\dot{\lambda} \frac{\mathbf{r}_{t}}{\left\|\mathbf{r}_{t}\right\|}$

where $\dot{\lambda}$ is obtained after eliminating the tangential reaction components from the slip rule:

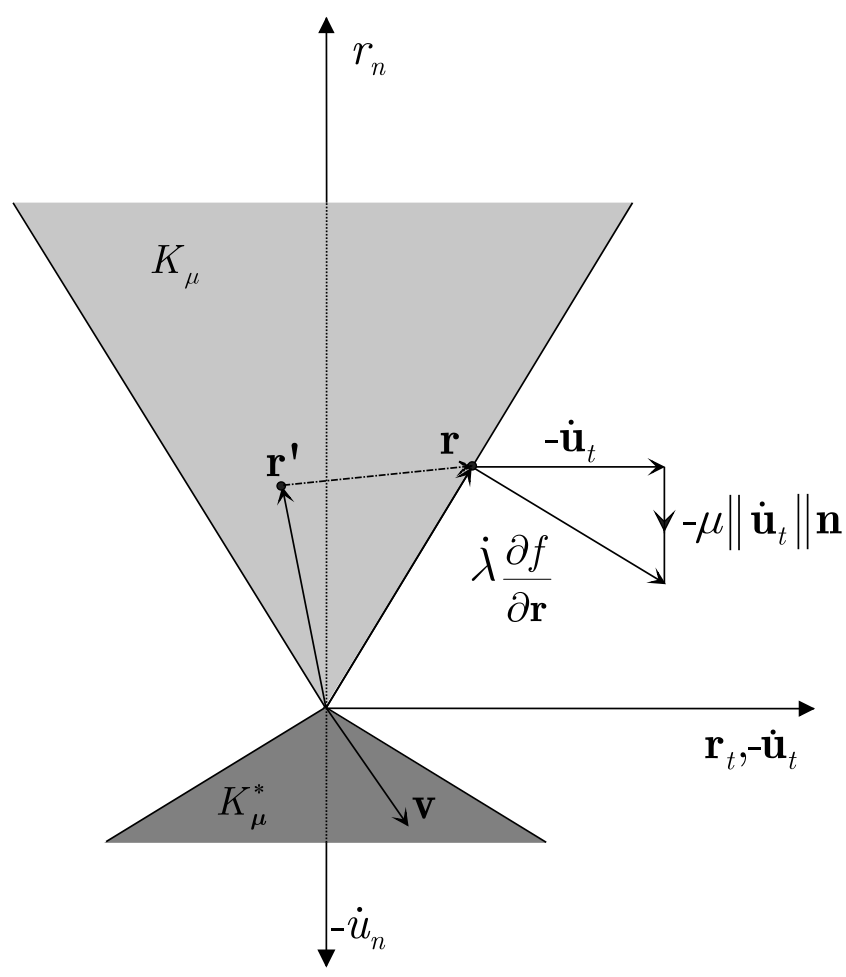

Fig. 4 Non-normality of the velocity vector

$\dot{\lambda}=\left\|-\dot{\mathbf{u}}_{t}\right\|$

Indeed the first relation of (32) yields back $-\dot{u}_{n}=0$, that is during sliding contact must hold. An obvious vector addition (see Fig. 4) gives :

$-\left(\dot{\mathbf{u}}_{t}+\left(\dot{u}_{n}+\mu \dot{\lambda}\right) \mathbf{n}\right)=\dot{\lambda} \operatorname{grad} f$

At the apex the friction criterion is not differentiable and the vector gradient should be replaced by any subgradient $\mathbf{v}$ as depicted in Fig. 4:

$-\left(\dot{\mathbf{u}}_{t}+\left(\dot{u}_{n}+\mu\left\|-\dot{\mathbf{u}}_{t}\right\|\right) \mathbf{n}\right)=\mathbf{v}$

Any vector $\mathbf{v}$ satisfying the following inequality, known as the convexity inequality for non-differentiable function, is a subgradient:

$f\left(\mathbf{r}^{\prime}\right) \geq f(\mathbf{r})+\left(\mathbf{r}^{\prime}-\mathbf{r}\right) \cdot \mathbf{v} \quad \forall \mathbf{r}^{\prime} \in \mathbb{R}^{3}$

The set of all subgradients is called subdifferential (see[3]), and is denoted by $\partial f(\mathbf{r})$. This more general definition allows to rewrite (34) as

$-\left(\dot{\mathbf{u}}_{t}+\left(\dot{u}_{n}+\mu\left\|-\dot{\mathbf{u}}_{t}\right\|\right) \mathbf{n}\right) \in \partial f(\mathbf{r})$

The subdifferential of a convex function defines a convex cone, called normal cone. Furthermore the subdifferential of the cone $K_{\mu}$ at the origin corresponds to its dual cone:

$\partial f(\mathbf{0})=K_{\mu}^{*}=\left\{\mathbf{v} \in \mathbb{R}^{3}: \mu\left\|\mathbf{v}_{t}\right\|+v_{n} \leq 0\right\}$

The relation (37) is valid everywhere on the friction surface and coincide with the (34) everywhere except at 
the origin $(\mathbf{r}=\mathbf{0})$. Therefore this relation corresponds to the frictional contact law. As it is well known from Convex Analysis, the normality rule (37) is equivalent to the following variational inequality:

$\mathbf{r} \in K_{\mu}: \quad\left(\mathbf{r}-\mathbf{r}^{\prime}\right) \cdot \mathbf{v} \geq 0, \quad \forall \mathbf{r}^{\prime} \in K_{\mu}$

where the components of the vector $\mathbf{v}$ are

$\mathbf{v}_{t}=-\dot{\mathbf{u}}_{t}, \quad v_{n}=-\left(\dot{u}_{n}+\mu\left\|-\dot{\mathbf{u}}_{t}\right\| \mathbf{n}\right)$

Again the vector $\mathbf{v}$ satisfying the inequality (39) is unique except at the apex $(\mathbf{r}=\mathbf{0})$ where this inequality becomes

$K_{\mu}^{*}=\left\{\mathbf{v} \in \mathbb{R}^{3}: \mathbf{r}^{\prime} \cdot \mathbf{v} \leq 0, \quad \forall \mathbf{r}^{\prime} \in K_{\mu}\right\}$

Combining the definition of the dual cone (22) and the definition of the vector $\mathbf{v}(40)$, we can see that at the origin $(\mathbf{r}=\mathbf{0})$, the non-penetration condition for contacting bodies is recovered. The slip rule in the form (37) is obtained for an anisotropic model by simply replacing " $\mu\left\|-\left(\dot{\mathbf{u}}_{t}\right)\right\|$ " with " $\left\|-\left(\dot{\mathbf{u}}_{t}\right)\right\|_{\mu}^{*}$.

\section{Discrete frictional contact law}

The inequality developed in the previous section is now discretized so it can be used in the local stage where the reaction are computed. The time interval $[0, T]$ is partitioned into $N$ sub-intervals of size $\triangle t$, not necessarily equal, according to

$0=t_{0}<t_{1}<\cdots<t_{n-1}<t_{n}<\cdots<t_{N}=T$

We set $\omega\left(t_{n}\right)$ and $\triangle \omega=\omega_{n}-\omega_{n-1}$, where $\omega$ represents any variable. Between two time instants, the velocity is assumed to be constant. In order to ensure convergence and stability requirements, the implicit scheme is considered. As a result of a backward-Euler type approximation of (39), the frictional contact law is satisfied at the end of each time step:

$\left\{\begin{array}{l}\mathbf{r}^{n+1} \in K_{\mu} \quad \text { such that } \\ \Delta \mathbf{v} \cdot\left(\mathbf{r}^{\prime}-\mathbf{r}^{n+1}\right) \leq 0, \quad \forall \mathbf{r}^{\prime} \in K_{\mu}\end{array}\right.$

where the components of the vector $\Delta \mathbf{v}$ are given by

$\Delta \mathbf{v}_{t}=-\Delta \mathbf{u}_{t}$ and $\Delta v_{n}=-\left(\Delta u_{n}+\left\|-\Delta \mathbf{u}_{t}\right\|_{\mu}^{*}\right)$

The previous inequality can be transformed into a projection inequality

$\left\{\begin{array}{l}\text { Find } \mathbf{r}^{n+1} \in K_{\mu} \quad \text { such that } \\ \left(\mathbf{r}^{n+1}-\tau\right) \cdot\left(\mathbf{r}^{\prime}-\mathbf{r}^{n+1}\right) \geq 0, \quad \forall \mathbf{r}^{\prime} \in K_{\mu}\end{array}\right.$

where the vector $\tau$ is given by

$\tau=\left\{\begin{array}{c}\tau_{t} \\ \tau_{n}\end{array}\right\}=\left\{\begin{array}{c}\mathbf{r}_{t}^{n+1}-\rho \Delta \mathbf{u}_{t} \\ r_{n}^{n+1}-\rho\left(\Delta u_{n}+\left\|\left(-\Delta \mathbf{u}_{t}\right)\right\|_{\mu}^{*}\right)\end{array}\right\}$

The last inequality means that the reaction at the end of the time step is the projection of the augmented surface traction $\tau$ onto the convex Coulomb's cone $K_{\mu}$ :

$\mathbf{r}^{n+1}=\operatorname{proj}\left(\tau, K_{\mu}\right)$
Three different situations emerge according to the position of the prediction in the forces space. The first case corresponds to a prediction located in the cone $K_{\mu}$. Its projection is the prediction itself, i.e. $\mathbf{r}^{n+1}=\tau$. The second one relates to a prediction situated in the cone $K_{\mu}^{*}$, where its projection turns out to be the origin of the forces space, i.e. $\mathbf{r}^{n+1}=\mathbf{0}$. In the last case, the prediction is neither in $K_{\mu}$ nor in $K_{\mu}^{*}$ and the corrector step requires computing the projection of the prediction. The projection of a point onto a convex set is equivalent to the minimization of the distance between this point and the convex set. In the present situation (orhtotropic friction condition), it leads to a quartic equation that has one positive root. The details of the derivation can be found in [1].

\section{Finite-step boundary value problem and variational formulation}

The solution of the elastic frictional-contact initial boundary value problem, under a given history of external actions, requires following the evolution of the body response since the frictional contact law is intrinsically path-dependant. A numerical technique, which combines a space and time discretization, is used to solve this problem. The time discretization is based on a subdivision of the external actions history into a sequence of loading conditions at selected time instants. The solution is then achieved by solving a sequence of problems in which the load increments are applied and the variables at the end of each increment are updated. We suppose that the solution of the problem is known at time $t_{n}$. Given the body force $\overline{\mathbf{f}}^{n+1}$, the surface traction $\overline{\mathbf{t}}^{n+1}$ and the imposed displacement $\overline{\mathbf{u}}^{n+1}$, it is required to solve the governing equations listed below:

- in $\Omega(0)$ :

$\operatorname{Div} \mathbf{P}^{n+1}+\overline{\mathbf{f}}_{0}^{n+1}=\mathbf{0}$

$$
\mathbf{E}^{n+1}=\frac{1}{2}\left(\mathbf{F}^{n+1}+\left(\mathbf{F}^{n+1}\right)^{\mathrm{T}}+\left(\mathbf{F}^{n+1}\right)^{\mathrm{T}} \mathbf{F}^{n+1}\right)
$$

$\mathbf{F}^{n+1}=\nabla \mathbf{u}^{n+1}$

$\mathbf{S}^{n+1}=\left.\frac{\partial W(\mathbf{E})}{\partial \mathbf{E}}\right|^{n+1}, \quad \mathbf{P}^{n+1}=\mathbf{F}^{n+1} \mathbf{S}^{n+1}$

- on $\Gamma_{t}(0)$ :

$\mathbf{P}^{n+1} \mathbf{n}_{0}=\overline{\mathbf{t}}_{0}^{n+1}$

- on $\Gamma_{u}(0)$ :

$\mathbf{u}=\overline{\mathbf{u}}^{n+1}$

- on $\Gamma_{c}(0)$ :

$\mathbf{r}^{n+1}=\operatorname{proj}\left(\boldsymbol{\tau}(\Delta \mathbf{u}), K_{\mu}\right)$ 
The standard approach to derive the principle of virtual work over an increment consists in taking the inner product of the local equilibrium equation (46) with a test function $\delta \mathbf{u}$ satisfying $\delta \mathbf{u}=0$ on $\Gamma_{u}$ and $\delta u_{n} \geq 0$ on $\Gamma_{c}$ (virtual displacement increment):

$$
\int_{\Omega(0)} \operatorname{Div} \mathbf{P}^{n+1} \cdot \delta \mathbf{u} \mathrm{d} \Omega+\int_{\Omega(0)} \overline{\mathbf{f}}_{0}^{n+1} \cdot \delta \mathbf{u} \mathrm{d} \Omega=0
$$

Integrating by parts the first term and taking into account the boundary conditions leads to the following variational equation:

$$
\begin{aligned}
& \int_{\Omega(0)} \mathbf{S}^{n+1} \cdot \delta \mathbf{E} \mathrm{d} \Omega-\int_{\Omega(0)} \overline{\mathbf{f}}_{0}^{n+1} \cdot \delta \mathbf{u ~ d} \Omega \\
& \quad-\int_{\Gamma_{t}(0)} \overline{\mathbf{t}}_{0}^{n+1} \cdot \delta \mathbf{u} \mathrm{d} \Gamma-\int_{\Gamma_{c}(0)} \mathbf{r}^{n+1} \cdot \delta \mathbf{u} \mathrm{d} \Gamma=0
\end{aligned}
$$

where the first Piola-Kirchhoff stress tensor $\mathbf{P}^{n+1}$ has been exchanged with the second Piola-Kirchhoff tensor $\mathbf{S}^{n+1}$. Taking into account that the stress filed is in equilibrium at $t_{n}$, the weak form can be written in terms of finite increments as follows:

$$
\begin{aligned}
& \delta\left\{\int_{\Omega(0)} \Delta W(\Delta \mathbf{E}) \mathrm{d} \Omega-\int_{\Omega(0)} \Delta \overline{\mathbf{f}}_{0} \cdot \Delta \mathbf{u} \mathrm{d} \Omega\right. \\
& \left.\quad-\int_{\Gamma_{t}(0)} \Delta \overline{\mathbf{t}}_{0} \cdot \Delta \mathbf{u} \mathrm{d} \Gamma-\int_{\Gamma_{c}(0)} \Delta \mathbf{r} \cdot \Delta \mathbf{u} \mathrm{d} \Gamma\right\}=0
\end{aligned}
$$

where $\Delta W(\Delta \mathbf{E})$ is given by

$$
\Delta W(\Delta \mathbf{E})=\frac{1}{2}(\Delta \mathbf{E})^{\mathrm{T}} \mathbf{D}(\Delta \mathbf{E})
$$

and $\Delta \mathbf{E}$ by

$\Delta \mathbf{E}\left(\mathbf{u}^{n}, \Delta \mathbf{u}\right)=\mathbf{E}\left(\mathbf{u}^{n}+\Delta \mathbf{u}\right)-\mathbf{E}\left(\mathbf{u}^{n}\right)$

If the behavior on contact surface is frictionless, the functional (52) becomes the classical energy functional:

$$
\begin{gathered}
\delta\left\{\int_{\Omega(0)} \Delta W(\Delta \mathbf{E}) \mathrm{d} \Omega-\int_{\Omega(0)} \Delta \overline{\mathbf{f}} \cdot \Delta \mathbf{u} \mathrm{d} \Omega\right. \\
\left.-\int_{\Gamma_{t}(0)} \Delta \overline{\mathbf{t}}_{0} \cdot \Delta \mathbf{u} \mathrm{d} \Gamma\right\}=0
\end{gathered}
$$

Then, the problem in not path-dependant anymore and the total variables are used instead of their increments.

\section{Finite element discretization}

The displacement increment field and the transformation gradient are approximated according to:

$\Delta \mathbf{u}=\mathbf{N}(\mathbf{X}) \Delta \mathbf{U}, \quad \Delta \mathbf{F}=\mathbf{B}(\mathbf{X}) \Delta \mathbf{U}$ where $\Delta \mathbf{U}$ is the unknown nodal displacement increment vector, $\mathbf{N}(\mathbf{X})$ is the matrix of polynomial shape functions and the matrix $\mathbf{B}(\mathbf{X})$ is given by

$\mathbf{B}(\mathbf{X})=\frac{\partial \mathbf{N}(\mathbf{X})}{\partial \mathbf{X}}$

The strain increment can be decomposed as

$\Delta \mathbf{E}=\left(\mathbf{B}_{\mathrm{L}}\left(\mathbf{U}^{n}\right)+\mathbf{B}_{\mathrm{NL}}(\Delta \mathbf{U})\right) \Delta \mathbf{U}$

The operator $\mathbf{B}_{\mathrm{L}}$ relates the linear part of the strain tensor to the nodal displacement vector and $\mathbf{B}_{\mathrm{NL}}$ relates the nonlinear part of the strain tensor to the nodal displacement vector. The compatibility conditions (50) on $\Gamma_{u}$ are enforced by substituting nodal unknowns by their corresponding values. Taking into account (58) and (59), the fully discrete form of the functional is given by

$$
\int_{\Omega(0)} \Delta W\left(\Delta \mathbf{E}\left(\mathbf{U}^{n}, \Delta \mathbf{U}\right)\right) \mathrm{d} \Omega-\Delta \mathbf{F}^{\mathrm{ext}} \cdot \Delta \mathbf{U}-\Delta \mathbf{R} \cdot \Delta \mathbf{U}
$$

where $\Delta \mathbf{F}^{\text {ext }}$ corresponds to the generalized nodal force increment vector

$\Delta \mathbf{F}^{\mathrm{ext}}=\int_{\Omega(0)} \mathbf{N}^{\mathrm{T}} \Delta \overline{\mathbf{f}}_{0} \mathrm{~d} \boldsymbol{\Omega}+\int_{\Gamma_{t}(0)} \mathbf{N}^{\mathrm{T}} \Delta \overline{\mathbf{t}}_{0} \mathrm{~d} \Gamma$

and $\Delta \mathbf{R}$ is the equivalent contact reaction increment vector at nodes. The equilibrium equations over a time step are given by

$\int_{\Omega(0)} \frac{\partial \Delta W\left(\Delta \mathbf{E}\left(\mathbf{U}^{n}, \Delta \mathbf{U}\right)\right)}{\partial \Delta \mathbf{U}} \mathrm{d} \Omega-\Delta \mathbf{F}^{\mathrm{ext}}-\Delta \mathbf{R}=\mathbf{0}$

where

$\frac{\partial \Delta W\left(\Delta \mathbf{E}\left(\mathbf{U}^{n}, \Delta \mathbf{U}\right)\right)}{\partial \Delta \mathbf{U}}=\mathbf{K}_{\mathrm{T}}$

Combining the structural equilibrium equations (62) with the incremental frictional contact constitutive relation (51), the solution of the boundary value problem over a time step is obtained by solving the following system of equations:

$\mathbf{K}_{\mathrm{T}} \Delta \mathbf{U}-\Delta \mathbf{F}^{\mathrm{ext}}-\Delta \mathbf{R}=\mathbf{0}$
$\mathbf{R}^{n+1}=\operatorname{proj}\left(\tau(\Delta \mathbf{U}), K_{\mu}\right) \quad$ on $\Gamma_{c}$

$\Delta \mathbf{U}=\Delta \overline{\mathbf{U}} \quad$ on $\Gamma_{u}$

\section{Solution algorithm}

The system of equations (63-65) has to be solved iteratively since the displacement increment vector, the contact surface and the reactions $\Delta \mathbf{R}$ are unknown. The solution algorithm (see Box 3) tackle separately the geometric and the contact nonlinearities. At the begin- 


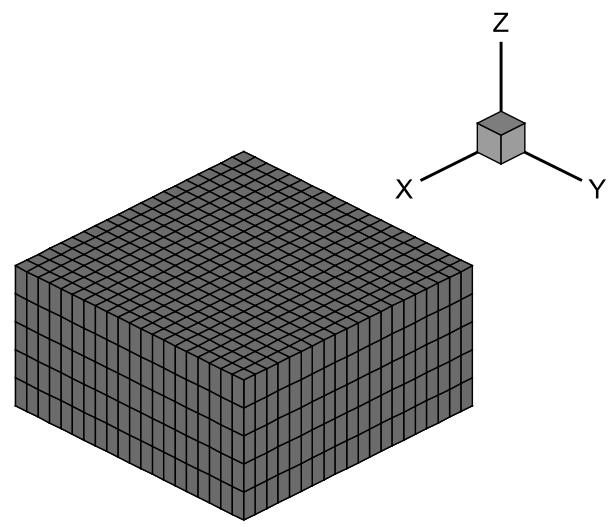

Fig. 5 Block mesh

FOR EACH LOAD STEP :

- Initialisation : $\Delta \mathbf{U}^{0}=\mathbf{0}$ and $\Delta \mathbf{S}^{0}=\mathbf{0}$

- Compute the external load vector : $\Delta \mathbf{F}^{e x t}$

- Detect contact node using a gap function

- Equilibrium Loop i : $1 \rightarrow \mathrm{n}$

Assemble the tangent stiffness matrix $\mathbf{K}_{\mathrm{T}}$

Modify $\mathbf{K}_{\mathrm{T}}$ for essential boundary conditions

Solve : $\Delta \mathbf{U}=\mathbf{K}_{\mathrm{T}}^{-1}\left(\Delta \mathbf{F}^{\text {ext }}+\Delta \mathbf{R}^{0}\right)$

Update : $\mathbf{U}=\mathbf{U}+\Delta \mathbf{U}$

Convergence? : $i=i+\mathbf{1}$

On $\Gamma_{c}:$ Correct $\Delta \mathbf{U}$ and $\Delta \mathbf{R}$ according to Box 4

Solve : $\Delta \mathbf{U}=\mathbf{K}_{\mathrm{T}}^{-1}\left(\Delta \mathbf{F}^{e x t}+\Delta \mathbf{R}^{0}\right)$

- Outputs : $\Delta \mathrm{E}, \Delta \mathrm{S}$

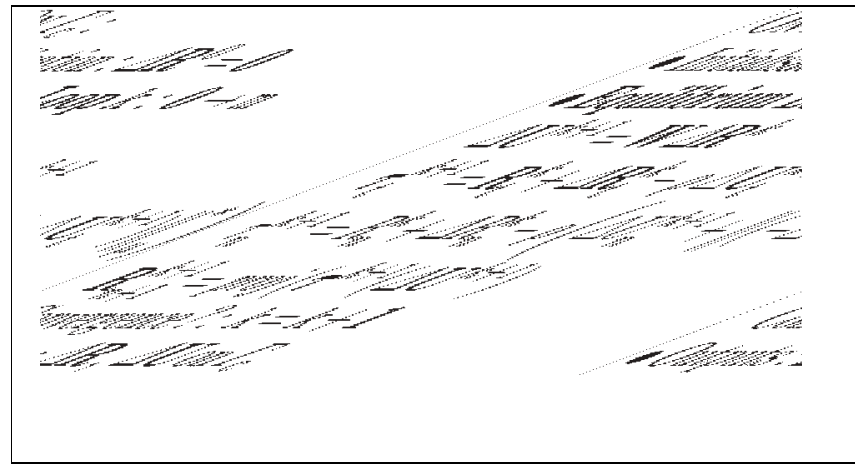

ning of each load step, $\Delta \mathbf{R}$ is set equal to zero and $\Delta \mathbf{U}$ is computed according to (63). Since the problem is geometrically nonlinear, the process is iterative. Now the reactions have to be computed and the penetration that has occurred by assuming $\Delta \mathbf{R}=\mathbf{0}$ must be corrected. This is achieved by applying the Uzawa iterative scheme as shown in Box 4. This procedure involves only variables associated with contact nodes. In contrast with classical methods (penalty, Lagrange multiplier), the present algorithm does not require an update of the global stiffness matrix during the contact iterations. Furthermore the total number of degree of freedom remain unchanged.

The displacement increments on the contact surface are computed using the flexibility matrix $\mathbf{W}$, defined in the local coordinate system:

$$
\mathbf{W}=\left[\begin{array}{lll}
w_{n n} & w_{n t_{x}} & w_{n t_{y}} \\
w_{n t_{x}} & w_{t_{x x}} & w_{n t_{x y}} \\
w_{n t_{y}} & w_{t_{x y}} & w_{t_{y y}}
\end{array}\right]
$$

Fig. 6 Contour plots of slip after compression

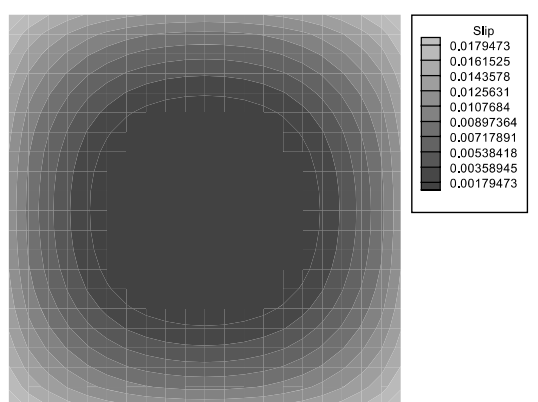

(a) $\mu_{x}=\mu_{y}=0.2$

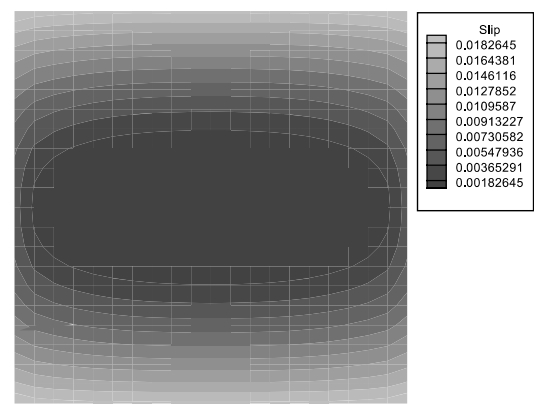

(b) $\mu_{x}=0.3, \mu_{y}=0.15$

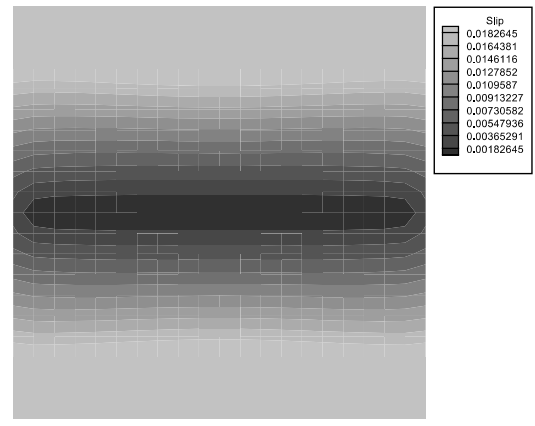

(c) $\mu_{x}=0.3, \mu_{y}=0.05$ 


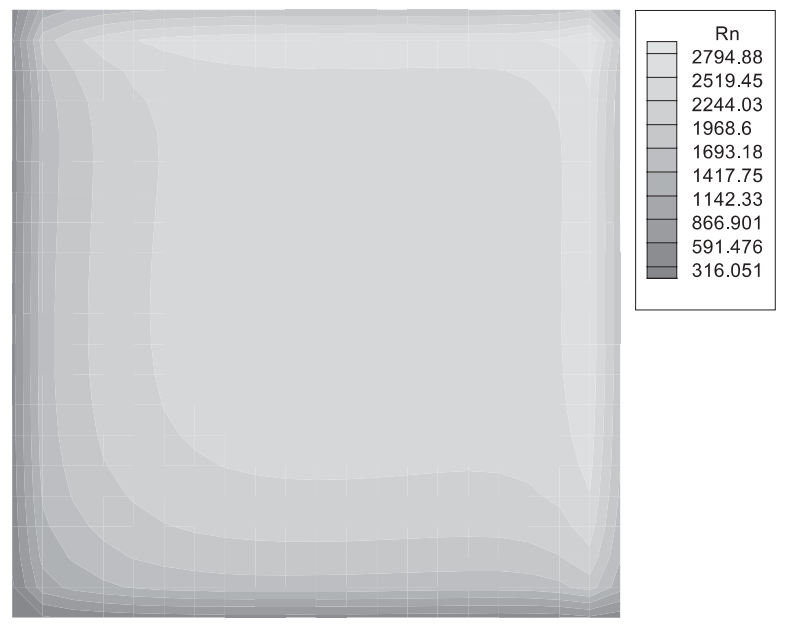

(a) $\mu_{x}=\mu_{y}=0.2$

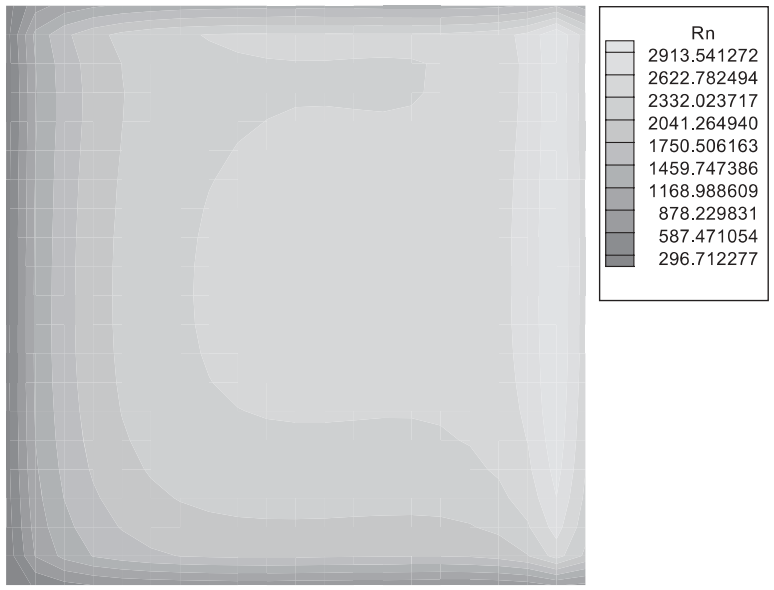

(b) $\mu_{x}=0.3, \mu_{y}=0.15$

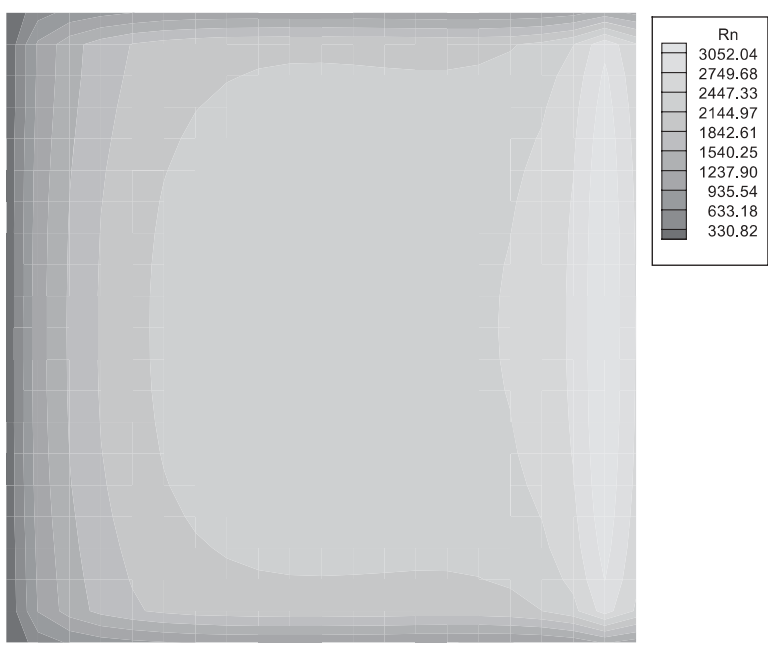

(c) $\mu_{x}=0.3, \mu_{y}=0.05$

Fig. 7 Contour plots of $r_{n}$ after compression

Next, the prediction $\tau$ is computed and projected on the cone $K_{\mu}$ to give the reaction (see Box 4). The Uzawa algorithm is known for being convergent but requiring quite a few iterations. The convergence rate strongly depends on the regularization factor $\rho$. A good choice is crucial to ensure that the algorithm will convergence within a few iterations. Our experience has shown that a different factor $\rho$ for each contact node gives a better convergence. In our calculations, the factor $\rho$ is calculated using the diagonal terms of the flexibility matrix:

$\rho=\frac{1}{\min \left(w_{n n}, w_{t_{x x}}, w_{t_{y y}}\right)}$

This choice has proven to be satisfactory for most cases.

\section{Numerical application}

The numerical algorithm developed above is used to study the motion of a block pushed on a frictional planar surface. The block is a rectangular prism with the following dimensions: base $=20 \mathrm{~mm} \times 20 \mathrm{~mm}$ and height $=10 \mathrm{~mm}$. The block is subdivided into 2000 eight-node brick-like elements as shown in Fig. 5. Each element has 27 integration points. The lower surface of the cube is in contact with a planar surface whose normal vector is $(0,0,1)$. The loading process involves 5 steps and is displacement-controlled. During the first step, the block is compressed by applying a uniform vertical displacement on the upper surface. The magnitude of the vertical displacement is $0.1 \mathrm{~mm}$. Next, the block is pushed horizontally by applying, in 4 steps, a uniform horizontal displacement on the upper surface. The direction of the horizontal motion is inclined at $45^{\circ}$ with respect to the $x$-axis. The magnitude of each horizontal displacement increment is $0.32 \mathrm{~mm}$. The block is assumed to be elastic with the following material properties:

- Young's modulus: $\mathrm{E}=210000 \mathrm{~N} / \mathrm{mm}^{2}$,

- Poisson's ratio: $=0.3$

The effect of frictional anisotropy is examined by considering three different sets of frictional coefficients $\mu_{x}$ and $\mu_{y}$ :

- Case 1: $\mu_{x}=\mu_{y}=0.2$, (isotropic)

- Case 2: $\mu_{x}=0.3$ and $\mu_{y}=0.15$,

- Case $3: \mu_{x}=0.3$ and $\mu_{y}=0.05$

The first case corresponds to the classical isotropic frictional model where the frictional behavior is identical for all sliding directions. A mild anisotropy is considered in the second case where the friction coefficient in the $x$-direction is twice the friction coefficient in the $y$-direction. In Case 3, the anisotropy is even stronger with a ratio $\mu_{x} / \mu_{y}$ equal to 6 .

\subsection{Compression of the block}

The frictional properties of the planar surface have a significant influence on the slip distribution. Figure 6 shows the contour plots of the slip for the three cases. The slip corresponds to the Euclidean norm of the 


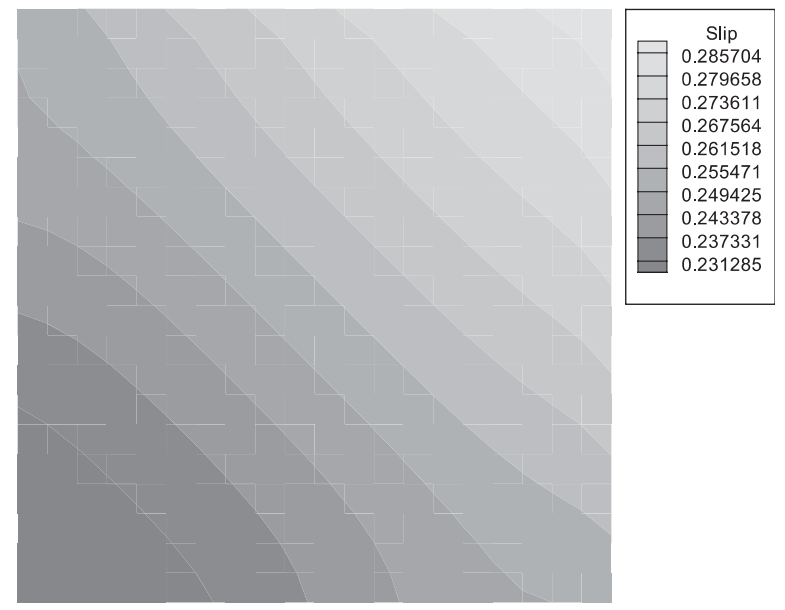

(a) $\mu_{x}=\mu_{y}=0.2$

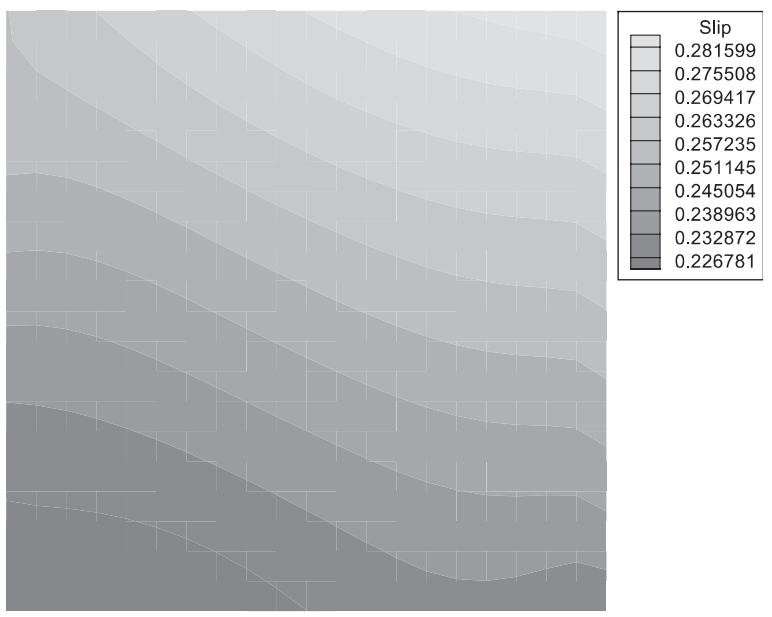

(b) $\mu_{x}=0.3, \mu_{y}=0.15$

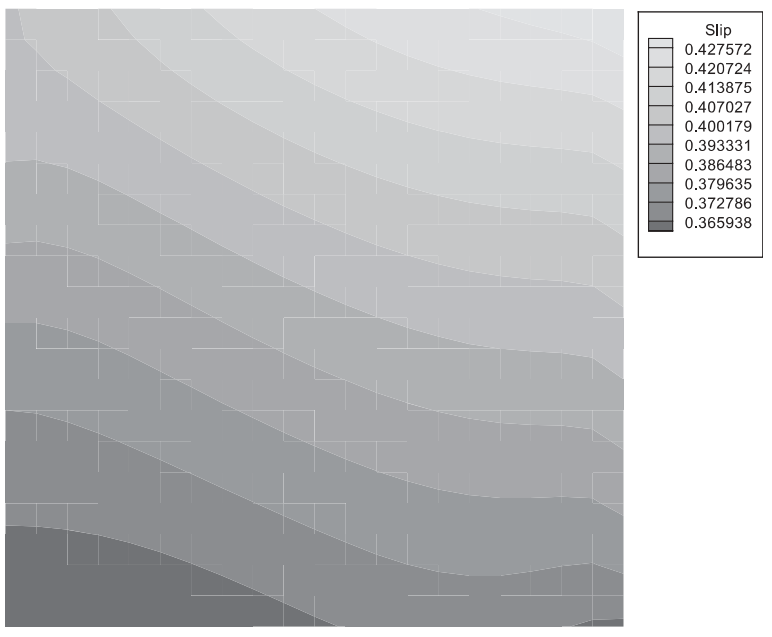

(c) $\mu_{x}=0.3, \mu_{y}=0.05$

Fig. 8 Contour plots of slip after sliding. a $\mu_{x}=\mu_{y}=0.2$ $\mu_{x}=0.3, \mu_{y}=0.15$ c $\mu_{x}=0.3, \mu_{y}=0.05$

tangential displacement of contact nodes. As expected, the stick zone is located around the base center and sliding increases monotonically as we get closer to the periphery. The shapes of the slip iso-values are similar to the friction criterion. The iso-values are circular for the isotropic case and elliptic for the anisotropic cases. The tangential displacement is obviously larger in the $y$-direction if the friction coefficient is smaller in this direction. The slip iso-values are flat ellipses for the strongly anisotropic model, exactly like the friction criterion. Contour plots of the normal reaction $r_{n}$ are shown in Fig. 7. The largest value of $r_{n}$ occurs around the base center but does not change notably with the frictional properties of the planar surface. The minimum of the normal reaction occurs at the periphery.

\subsection{Sliding motion of the block}

Figure 8 shows contour plots of the total slip at the end of the loading process. The maximum value of the sliding occurs at the front corner (with respect to motion). There is no significant difference between the value of the maximum slip for the isotropic model and the mildly anisotropic one. A larger displacement in the $y$-direction is observed for the strongly anisotropic model as a result of a low friction coefficient in this direction. The contour plots of the normal reaction at the end of the loading are shown in Fig. 9. Again, the maximum and the minimum of $r_{n}$ are very close for all models. The maximum occurs in the "front" area while the minimum occurs in the "back" area. The deformed meshes for the three models are shown in Fig. 10. The motion of the upper face is imposed but the motion of the lower face depends strongly on the frictional properties of the planar surface. If the frictional behavior is independent of the sliding direction (isotropic model), the lower and the upper faces of the block move in the same direction. Therefore no twist (distortion due to different motion directions for the lower and upper faces) of the cube occurs. However, deformation due to the relative displacement between the upper and the lower faces takes place. The amplitude of this deformation depends on the elastic properties of the cube. On the other hand if an anisotropic frictional model is considered then the motion direction of the lower face does not coincide with the motion direction of the upper face and the block is twisted. The magnitude of this distortion depends on the friction coefficients and the elastic properties. The distortion is stronger for the strongly anisotropic model.

\section{Conclusion}

In this paper, the motion of a deformable body sliding on a half-plane has been investigated. Large displacements but small strains have been considered. The frictional behavior has been modelled by an elliptic cone. The algorithm used to solve the problem is based on a weak variational statement of the frictional contact law. An uncoupled strategy was used to solve the discrete equations. The study shows that frictional properties 
Fig. 9 Contour plots of $r_{n}$ after sliding
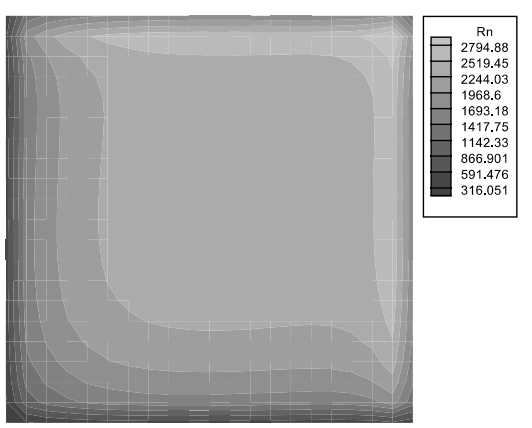

(a) $\mu_{x}=\mu_{y}=0.2$

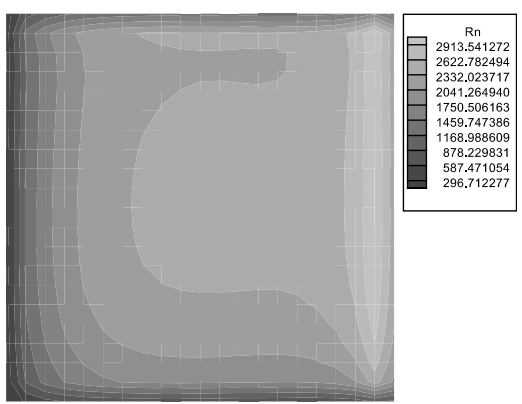

(b) $\mu_{x}=0.3, \mu_{y}=0.15$

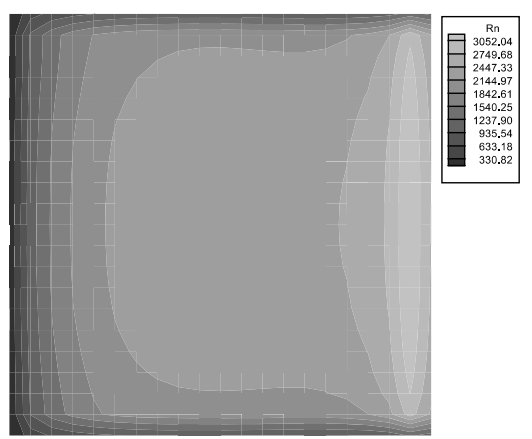

(c) $\mu_{x}=0.3, \mu_{y}=0.05$

Fig. $103 \mathrm{D}$ deformed mesh after sliding

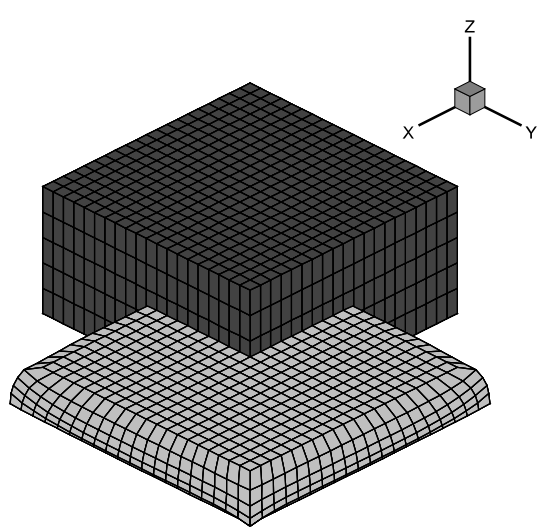

(a) $\mu_{x}=\mu_{y}=0.2$

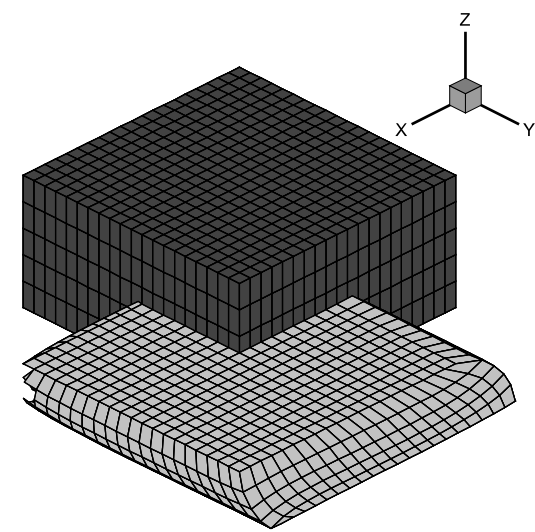

(b) $\mu_{x}=0.3, \mu_{y}=0.15$

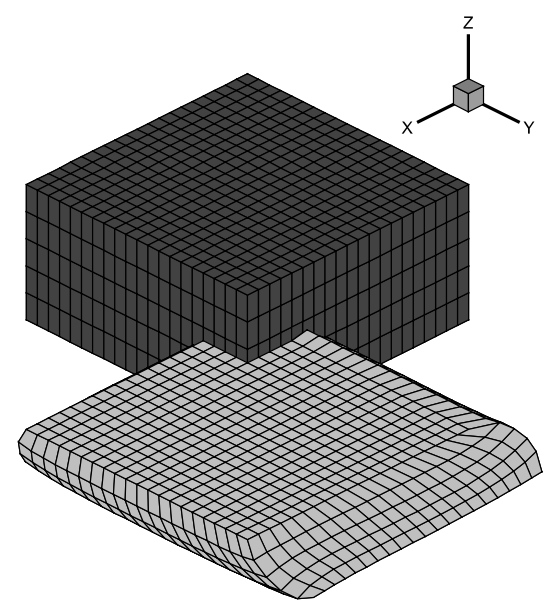

(c) $\mu_{x}=0.3, \mu_{y}=0.05$ 
have a strong influence on the trajectory of a deformable body sliding on a frictional surface.

\section{References}

1. Hjiaj M, Feng ZQ, de Saxcé G, Mróz Z. (2004) Three-dimensional finite element computations for frictional contact prob- lems with non-associated sliding rule. Int. J. Numer. Meth. Eng. 60: 2045-2076

2. Michałowski R, Mróz Z. (1978) Associated and non-associated sliding rules in contact friction problems. Arch. Mech. 30: 259276

3. Rockafellar RT, Wets J-B. (1998) Variational analysis. Springer, Berlin, Heidelberg New York

4. Wriggers P. (2002) Computational contact mechanics. Wiley, New York 